\title{
Wavelet Compression of ECG Signals by JPEG2000
}

\author{
Ali Bilgin ${ }^{1}$, Michael W. Marcellin ${ }^{1}$, and Maria I. Altbach ${ }^{2}$ \\ ${ }^{1}$ Dept. of Electrical and Computer Engineering, The University of Arizona, Tucson, AZ 85721 \\ ${ }^{2}$ Dept. of Radiology, The University of Arizona, Tucson, AZ 85724
}

JPEG2000 is the latest international standard for compression of still images. Although the JPEG2000 codec is designed to compress images, we illustrate that it can also be used to compress other signals. As an example, we illustrate how the JPEG2000 codec can be used to compress electrocardiogram (ECG) data. Experiments using the MIT-BIH arrhythmia database illustrate that the proposed approach outperforms many existing ECG compression schemes. The desirable characteristics of the JPEG2000 codec, such as precise rate control and progressive quality, are retained in the presented scheme.

To compress the ECG data using a JPEG2000 codec, the one-dimensional ECG sequence is processed to produce a two-dimensional matrix. Since it is desirable to exploit both the intrabeat and interbeat dependencies, we first separate each "period" of the ECG. Each period is normalized to account for their differences in length and stored as one row of a matrix. This matrix is then encoded using JPEG2000.

In Table 1, the proposed method is compared to other methods in the literature for different compression ratios (CR) and records. The methods in this table include other wavelet-based coders, as well as the parametric ECG signal coder ASEC [3]. The results indicate that the proposed method compares favorably with existing ones.

Table 1: Percent root mean square difference (PRD) comparison of different algorithms.

\begin{tabular}{|c|c|c|c|}
\hline Algorithm & Record & CR & PRD(\%) \\
\hline Lu et. al [4] & 117 & $8: 1$ & 1.18 \\
\hline Hilton [2] & 117 & $8: 1$ & 2.6 \\
\hline Djohan et. al [1] & 117 & $8: 1$ & 3.9 \\
\hline Proposed & 117 & $8: 1$ & 0.86 \\
\hline Wei et. al [5] & 117 & $10: 1$ & 1.18 \\
\hline Proposed & 117 & $10: 1$ & 1.03 \\
\hline \hline ASEC [3] & 119 & $21.6: 1$ & 5.5 \\
\hline Lu et. al [4] & 119 & $21.6: 1$ & 5.0 \\
\hline Proposed & 119 & $21.6: 1$ & 3.76 \\
\hline
\end{tabular}

\section{REFERENCES:}

[1] A. Djohan, T. Q. Nguyen, and W. J. Tompkins, "ECG compression using discrete symmetric wavelet transform," in Proc. of $17^{\text {th }}$ Int. IEEE Med. Biol. Conf., 1995.

[2] M. L. Hilton, "Wavelet and wavelet packet compression of electrocardiograms", IEEE Trans. on Biomedical Engineering, vol. 44, pp. 394-402, May 1997.

[3] Y. Zigel, A. Cohen, A. Abu-ful, A. Wagshal, and A. Katz, "Analysis by synthesis ECG signal compression”, Comput. in Cardiology, vol. 24, pp. 279-282, 1997.

[4] Z. Lu, D. Y. Kim, and W. A. Pearlman, "Wavelet compression of ECG signals by the set partitioning in hierarchical trees algorithm", IEEE Trans. on Biomedical Engineering, vol. 47, pp. 849-856, July 2000.

[5] J.-J. Wei, C.-J. Chang, N.-K. Chou, and G.-J. Jan, "ECG data compression using truncated singular value decomposition", IEEE Trans. on Information Technology in Biomedicine, vol. 5, pp. 290-299, Dec. 2001.

An extended version of this work can be found in A. Bilgin, M. W. Marcellin, M. I. Altbach, "Compression of ECG Signals using JPEG2000,” IEEE Transactions on Consumer Electronics, November 2003. 